\title{
Influence of doping profiles on coherent acoustic phonon detection and generation in semiconductors
}

\author{
F. Hudert, ${ }^{1, a)}$ A. Bartels, ${ }^{1}$ T. Dekorsy, ${ }^{1}$ and K. Köhler ${ }^{2}$ \\ ${ }^{1}$ Department of Physics and Center for Applied Photonics, University of Konstanz, D-78457 Konstanz, \\ Germany \\ ${ }^{2}$ Fraunhofer-Institut für Angewandte Festkörperphysik, D-79108 Freiburg, Germany
}

(Received 28 May 2008; accepted 14 October 2008; published online 17 December 2008)

\begin{abstract}
The doping profile in different $n$-doped GaAs homoepitaxial structures grown by molecular beam epitaxy is investigated in the time domain by employing a laser based picosecond ultrasound technique in a contactless and noninvasive way. Experiments based on asynchronous optical sampling employ two femtosecond lasers, which allow us to detect changes in the optical reflectivity over a $1 \mathrm{~ns}$ time delay with a signal-to-noise ratio of $10^{7}$ and $100 \mathrm{fs}$ time resolution in $<1 \mathrm{~min}$ of acquisition time. We show that the doping profile with doping densities of the order of $10^{18} \mathrm{~cm}^{-3}$ can be detected with picosecond ultrasound, although there is no difference in the acoustic properties of the doped and undoped region. The detection mechanism is based on a different sensitivity function for a coherent strain pulse in the doped and undoped regions. These results are corroborated by experiments at room temperature and $10 \mathrm{~K}$.
\end{abstract}

\section{INTRODUCTION}

Laser based picosecond ultrasonics has been implemented in the study of thin films and heterostructures over the past 20 years. ${ }^{1}$ With a growing understanding of laser induced strain pulse propagation, a wide range of spectroscopic applications is emerging. Prominent examples are phonon cavities, ${ }^{2}$ light modulators based on optoelastic interaction, ${ }^{3}$ and phononic crystals. ${ }^{4}$ Laser based picosecond ultrasonics provides an excellent tool for a noninvasive subsurface characterization of nanostructures as shown in recent works. $^{5-7}$

In order to characterize a heterostructure, usually a picosecond acoustic pulse is created by absorption of a subpicosecond laser pulse in a thin metal layer on the surface of the sample. ${ }^{8}$ The acoustic pulse propagation through the sample is then monitored via the changes in reflectivity or transmissivity mediated through acousto-optical coupling. Since the acoustic propagation is very sensitive to impedance mismatches at interfaces of different materials, information on, for example, film thicknesses and interface properties can be retrieved from the reflectivity $(\Delta R)$ or transmission $(\Delta T)$ transients. However, not only structural information associated with impedance mismatches is accessible by this technique. Lin et al. ${ }^{9}$ showed results where piezoelectric generation of picosecond acoustic pulses in a $p$ - $n$ junction leads to information on the location and width of the depletion zone of the $p$ - $n$ junction without the need for an impedance mismatch in a strongly piezoelectric material such as GaN.

Here we show the possibility to characterize doping profiles that are not associated with impedance mismatches in a nonpiezoelectric material of high technological importance, i.e., GaAs. Experiments are performed on (100) and (111) GaAs samples at room temperature and $10 \mathrm{~K}$. We will show

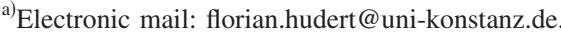

that information on the doping profile can be obtained by exploiting the spatially inhomogeneous generation of the strain pulse and the dependence of the optical constants on the doping concentration. ${ }^{10}$

\section{EXPERIMENTAL}

We have used a recently introduced optical pump-probe technique called asynchronous optical sampling (ASOPS). ${ }^{11}$ 2 fs passively mode-locked Ti:sapphire lasers with repetition rates of about $1 \mathrm{GHz}$ are used to generate the pump and the probe pulses of $<100$ fs duration. The repetition rates of both lasers are linked to each other by an active feedback in such a way that the pump laser has a repetition rate that is 10 $\mathrm{kHz}$ higher than the repetition rate of the probe laser. Thus, the relative time delay between pump and probe pulse is automatically scanned through the entire measurement window with a difference frequency of $10 \mathrm{kHz}$. The length of the measurement window is given by the inverse of the repetition rate and extends over $1 \mathrm{~ns}$. The advantages of the ASOPS technique are that (i) it does not require a mechanical delay line, (ii) it allows for a high signal accumulation rate, (iii) it provides online monitoring of the signal, and (iv) it is free from pointing instabilities associated with delay lines. In addition both lasers are individually tunable between 750 and $860 \mathrm{~nm}$, which allows for two color pump probe spectroscopy. Low temperature measurements were performed using a He flow cryostat (Oxford Microstat).

The samples studied in this paper consist of $n$-doped GaAs films of $1200 \mathrm{~nm}$ thickness that were grown on GaAs substrates via molecular beam epitaxy. The nominal doping concentrations are $2 \times 10^{18} \mathrm{~cm}^{-3}$. Samples were grown on (100) and (111) oriented substrates. As a reference, we used a sample consisting of an epitaxially grown $500 \mathrm{~nm}$ thick undoped GaAs film on top of a (100) oriented GaAs substrate. 


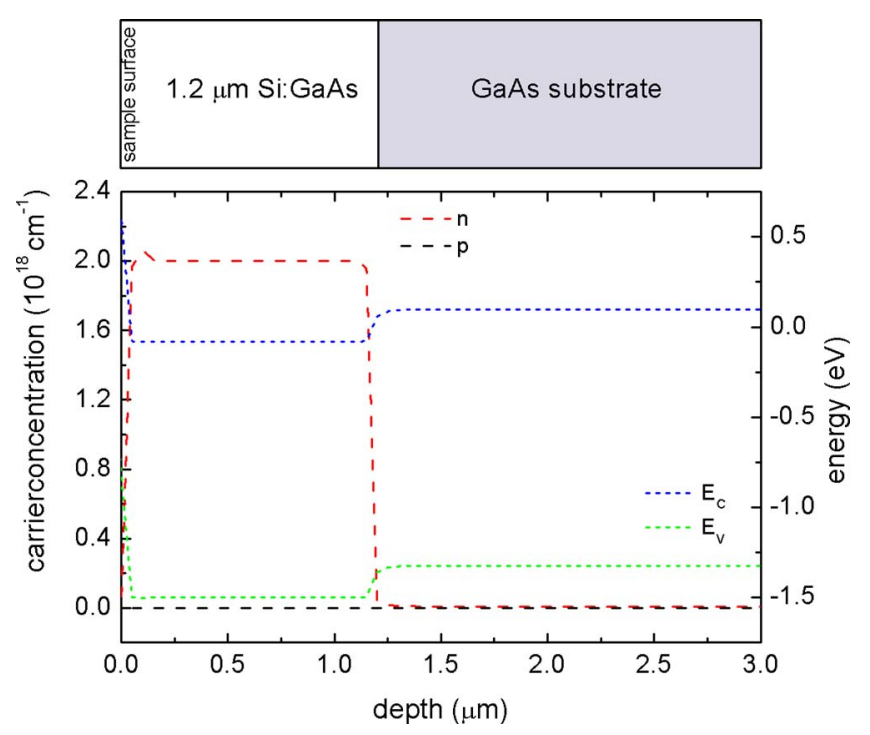

FIG. 1. (Color online) Schematical representation of the sample's geometry and calculated doping concentrations ( $n$ for electrons and $p$ for holes) in addition to conduction and valence bands ( $E_{C}$ and $E_{V}$, respectively) of a doped sample assuming a background doping concentration of the GaAs substrate of $1 \times 10^{16} \mathrm{~cm}^{-3}$.

A reflection geometry is used in the experimental setup. Pump and probe beams are aligned at about $1 \mathrm{~cm}$ parallel to each other and focused onto the sample through the same lens. Pump and probe beams impinge onto the sample at an angle of $<3^{\circ}$. Both beams are cross polarized and a polarizing beam splitting cube in front of the detector is used to block scattered pump light. For these measurements average pump and probe powers of about 400 and $40 \mathrm{~mW}$, respectively, were used. The spot sizes were measured to be about $50 \mu \mathrm{m}$.

Figure 1 schematically shows the sample's geometry and the calculated depth profile of the carrier concentrations (electron concentration $n$ and hole concentration $p$ ) and the conduction and valence bands ( $E_{C}$ and $E_{V}$, respectively) for one of the doped samples, assuming a background doping concentration for the GaAs substrate of $1 \times 10^{16} \mathrm{~cm}^{-3}$. The band diagram is calculated with a one dimensional Poisson equation assuming full ionization of the dopants. The interface between the doped and the undoped region of the sample has a thickness of about $50 \mathrm{~nm}$, being limited by the Debye length. The large doping concentration in the top layer leads to a Burstein-Moss shift of about $120 \mathrm{meV}$ that is counterbalanced by band gap renormalization. ${ }^{12}$ These effects lead to a shift in the absorption threshold by $\sim 30 \mathrm{me} V$ for a doping concentration of $2 \times 10^{18} \mathrm{~cm}^{-3} \cdot{ }^{13}$ Hence the absorption coefficient at, e.g., $1.48 \mathrm{eV}$ at room temperature is lowered by a factor of $4 .{ }^{14}$

Figure 2 shows a typical transient of the changes in the reflectivity for the undoped reference sample measured with a pump and probe wavelength of $800 \mathrm{~nm}$. The dominating feature during the first picoseconds is a result of the electronic dynamics during the thermalization and energy relaxation of the hot carriers created by the pump pulse. On a 100 ps time scale the decay of the electronic signal is given by diffusion, bulk and surface recombination, and transport in

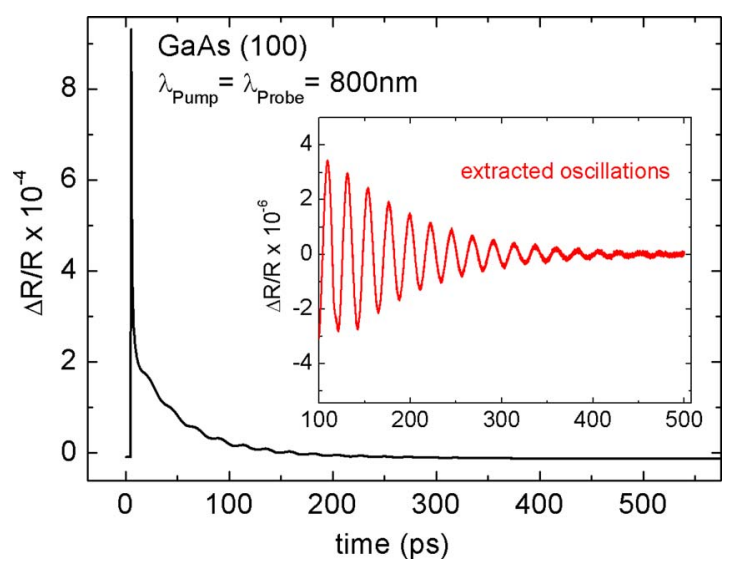

FIG. 2. (Color online) $\Delta R / R$ of the undoped reference sample measured at room temperature with a probe and pump wavelength of $800 \mathrm{~nm}$. The inset shows the oscillations of the reflectivity in the range of 100-500 ps.

space charge layers. ${ }^{15}$ The electronic dynamics, which induces a reflectivity change of the order of $10^{-4} \Delta R / R_{0}$, will be ignored throughout this paper.

We focus on the long living oscillations superimposed on the electronic contribution to the signal, which can be explained in the following way: the absorption of the pump light leads to an isotropic stress, a combination of both thermal stress and electronic stress due to the deformation potential. ${ }^{16}$ Subsequently, strain pulses are launched either at free surfaces or compositional interfaces or, as in this case, at the interface between the doped and the undoped region because of the inhomogeneous strain distribution as a result of the absorption profile. In the (111) oriented sample piezoelectric effects should also contribute to the strain pulse generation. However in contrast to Ref. 17, no definitive evidence of this could be found here. The (100) samples are not piezoelectrically active.

These plane strain pulses modulate the refractive index of the material leading to minute partial reflection of the probe light. The situation can be understood like an interferometer with one reflecting plane being the sample surface and the other one the propagating strain pulse. ${ }^{1}$ The reflected light will interfere constructively or destructively depending on the position of the strain pulse, leading to an oscillation of the reflected light intensity with a frequency $\nu$ defined by

$$
\nu=\frac{2 n}{\lambda_{\text {probe }}} v_{\text {sound }},
$$

where $n$ is the refractive index (probe refractive index: $n$ $=3.76), \lambda_{\text {probe }}$ is the wavelength of the probe light, and $v_{\text {sound }}$ is the longitudinal sound velocity in the material. This relation stems from the fact that the period of the oscillation, e.g., the time between completely destructive and completely constructive interferences, is equal to the time it takes for the strain pulse to advance half a wavelength of the probe light.

The inset of Fig. 2 shows these oscillations that were extracted by subtracting the electronic contribution through a moving average from the original signal. Exponentially decaying oscillations without any phase shifts are observed. In this simple case, a plane strain pulse is launched from the surface and propagates into the sample. The oscillations of 

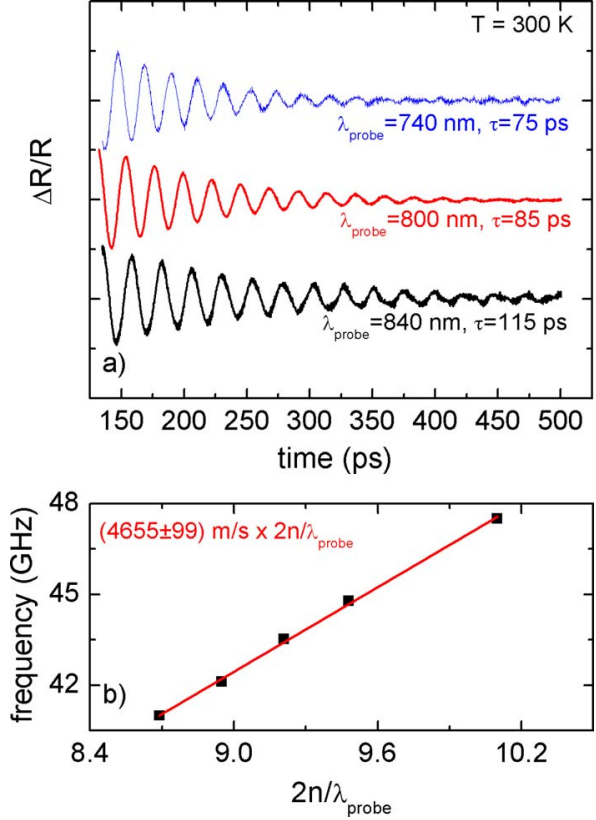

FIG. 3. (Color online) Results from a measurement series with varying probe wavelengths and constant pump wavelength for the undoped reference sample. (a) shows extracted oscillations for the probe wavelengths of 740 , 800 , and $840 \mathrm{~nm}$ in the range of $150-500 \mathrm{ps}$. (b) shows spectral analysis of the data in (a) showing the frequency of the oscillations over $2 n / \lambda_{\text {probe }}$.

the order of $10^{-6}$ in the $\Delta R / R$ signal are a result of the abovementioned interference of the probe light reflected from the surface and the probe light reflected from the strain pulse.

Figure 3(a) shows the extracted oscillations for three transients acquired with three different probe wavelengths (740, 800, and $840 \mathrm{~nm})$ and constant pump wavelength $(800$ $\mathrm{nm}$ ) for the same undoped reference sample. The oscillations of the three transients differ in their frequency and exhibit a different decay time ranging between $75 \mathrm{ps}$ at $\lambda_{\text {probe }}$ $=740 \mathrm{~nm}$ and $115 \mathrm{ps}$ at $\lambda_{\text {probe }}=840 \mathrm{~nm}$. The decay is dominated by the propagation of the strain pulse out of the penetration depth of the probe light. At $840 \mathrm{~nm}$ the probe light penetrates deeper into the sample depth $\left(\alpha_{840}^{-1} \mathrm{~nm}\right.$ $\approx 1000 \mathrm{~nm}$ ), thus leading to a larger decay time of the acoustic oscillation as compared to $740 \mathrm{~nm} \quad\left(\alpha_{740}^{-1} \mathrm{~nm}\right.$ $\approx 570 \mathrm{~nm}$ ).

Figure 3(b) shows the result of the spectral analysis of a series of pump probe measurements performed with different probe wavelengths, partly shown in Fig. 3(a). The red line is the result of a linear fit from Eq. (1) to the experimental data, and the resulting sound velocity of $4655 \pm 99 \mathrm{~m} / \mathrm{s}$ agrees well with the literature value for (100) GaAs ( $v_{\text {sound }}$ $=4730 \mathrm{~m} / \mathrm{s}$ ).

The results for the samples with the doped GaAs film on top of the substrate show a very different behavior. Figure 4 shows the extracted oscillations for the (100) sample measured at (a) $10 \mathrm{~K}$ and at (b) $300 \mathrm{~K}$ for two different probe wavelengths, namely, 795 and $840 \mathrm{~nm}$. The pump pulse wavelength in all cases is $800 \mathrm{~nm}$. All the transients exhibit a distinct and abrupt phase shift at $254 \mathrm{ps,} \mathrm{which} \mathrm{is} \mathrm{repeated}$ (more pronounced at $10 \mathrm{~K}$ ) after twice that time, 508 ps. 254 ps matches exactly the time a strain pulse needs to travel once through $1200 \mathrm{~nm}$ of (100) GaAs.

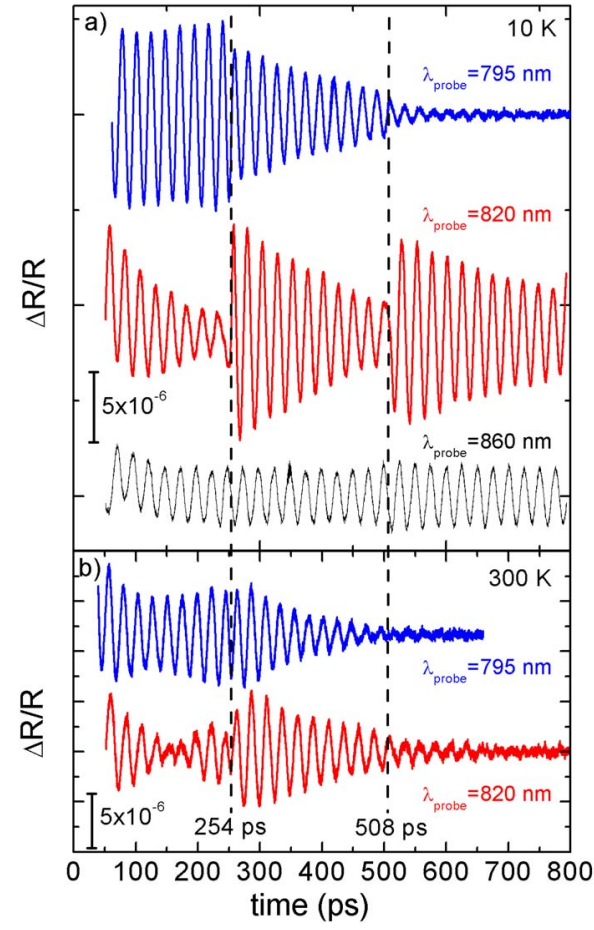

FIG. 4. (Color online) $\Delta R / R$ results for the doped GaAs (100) sample at (a) $10 \mathrm{~K}$ and (b) $300 \mathrm{~K}$. At both temperatures measurements were performed with a pump wavelength of $800 \mathrm{~nm}$ and probe wavelengths of 795 and 840 $\mathrm{nm}$

At $10 \mathrm{~K}$ and a probe wavelength of $840 \mathrm{~nm}$ the oscillations are still clearly visible at the end of the measurement window, which is due to the fact that at these temperatures the wavelength of $840 \mathrm{~nm}$ lies beneath the energy gap of GaAs $(816 \mathrm{~nm}$ at $10 \mathrm{~K})$ and thus, the sample is almost transparent for the probe light allowing for a detection of the strain pulse very deep into the sample. At the probe wavelength of $795 \mathrm{~nm}$ and at higher temperatures (at room temperature the band gap lies at $870 \mathrm{~nm}$ ), the phonon oscillations disappear much earlier due to the limited penetration depth of the probe light. Interestingly, no phase shift is observed at the below-band gap probe energy. This fact will be discussed later.

The (111) sample yields a similar behavior as depicted in Fig. 5, where the extracted oscillations for the (111) sample measured at (a) $10 \mathrm{~K}$ and at (b) $300 \mathrm{~K}$ for two different probe wavelengths, namely, 800 and $820 \mathrm{~nm}$, are shown. The pump wavelength in this case is again $800 \mathrm{~nm}$. The phase shifts for this sample occur after $225 \mathrm{ps}$, which is faster than for the (100) sample. This is due to a higher sound velocity in the (111) direction $\left(v_{\text {sound }}=5380 \mathrm{~m} / \mathrm{s}\right)$ than in the (100) direction $\left(v_{\text {sound }}=4730 \mathrm{~m} / \mathrm{s}\right)$ of the crystal.

The phonon oscillations in the (111) sample decay faster than that for the (100) sample at the given wavelengths due to the higher sound velocity. Hence a second phase shift at 450 ps cannot be resolved.

\section{DISCUSSION}

The most salient features of the observed transients are explained by the following model. In the vicinity of the fundamental band gap the absorption profile becomes inhomo- 


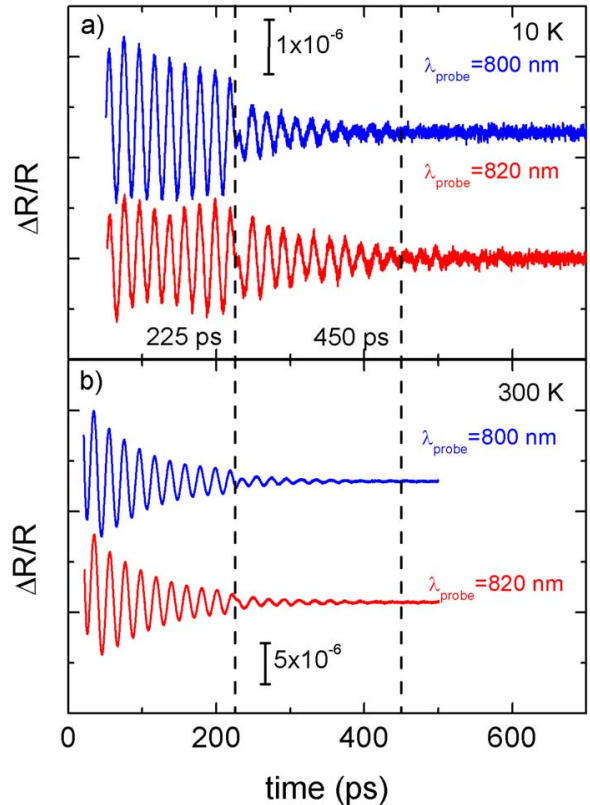

FIG. 5. (Color online) $\Delta R / R$ results for the doped GaAs (111) sample. Measurements were performed at (a) $10 \mathrm{~K}$ and (b) $300 \mathrm{~K}$ with a pump wavelength of $800 \mathrm{~nm}$. The probe wavelengths for the two transients in each graph were 800 and $820 \mathrm{~nm}$, respectively.

geneous due to the doping profile. In the doped region the absorption is lowered by the Burstein-Moss effect. Hence one strain pulse is launched from the sample's surface and a second strain pulse is launched from the $n-i$ interface. A second effect is the change in the sensitivity function for the strain pulse due to the doping profile. Although the strain pulse will pass the $n-i$ interface unaffected, the photoelastic modulation will be different. Since the unperturbed absorption and refractive index are changed by doping, the modulation due to the strain pulse will also be different. The time delay between $0 \mathrm{ps}$ and the first phase shift is given by two counter propagating strain pulses. The first phase shift appears when the pulse from the surface reaches the $n-i$ interface and vice versa. The second phase shift appears when the strain pulse generated at the $n-i$ interface has undergone a round trip to the surface.

The fact that the below-gap transient does not show a pronounced first phase shift suggests that the dominant contribution to the first phase shift stems from the strain pulse launched at the surface, which enters the $n-i$ interface and not by the strain pulse created at the $n-i$ interface, which reaches the surface.

In order to determine the depth resolution for the doping interface, we take a closer look at the observed phase shifts. Figure 6(a) shows a zoom into the region of the first phase shift for one transient of the GaAs (100) sample and one of the GaAs (111) sample. The time axis of both graphs is transformed into a depth using the respective sound velocities. This rescaling allows us to compare the sharpness of the phase shift with the calculated doping profile, which is shown in the lower part of Fig. 6. For both cases, one can see that the "duration" of the phase shift agrees very nicely with the length of the transition between doped and undoped regions. Where extractable, the duration of the second phase shift agrees very well with the first one.

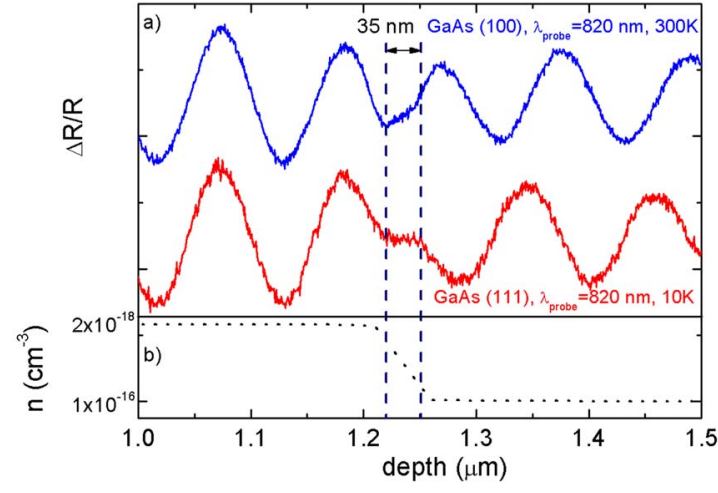

FIG. 6. (Color online) Part (a) of this graph shows a zoom into two transients from Figs. 3 and 4 ( $\lambda_{\text {probe }}=820 \mathrm{~nm}$ for both cases $)$ focusing on the region of the first phase shift. Part (b) shows a zoom into the spatial carrier distribution from Fig. 1. The time axis of the two transients is converted to depth using the sound velocity obtained from Fig. 2.

Although the exact shape and duration of the acoustic pulse remain unknown, an upper limit of about 30 ps surely can be assumed since the transition region in the transient is not wider than the calculated transition zone of the interface. It should also be noted that the abruptness of the transition is strongly influenced by the relatively high doping levels in the investigated samples. An analysis of a series of measurements at different positions of the sample yields a deviation in the position of the transition zone of about $5 \mathrm{~nm}$.

Another interesting feature of the results can be observed in the low temperature measurements in Fig. 4. The transient for a probe wavelength of $\lambda_{\text {probe }}=820 \mathrm{~nm}$ exhibits a strong increase in the oscillation amplitude after each phase shift. This can be explained by the fact that in this case the probe energy lies very close to the band gap energy of the undoped GaAs so that the elasto-optic coupling between the strain and the refractive index is enhanced. This leads to a larger sensitivity of the optical response to the strain pulse each time it enters the undoped region. Additionally, the superposition of oscillatory signals, which stem from different strain pulses propagating in the sample, may lead to an enhancement or phase shift in these signals. The assignment of these effects requires a numerical modeling of the pulse propagation and the optical response of the sample. ${ }^{18,19}$

\section{SUMMARY}

We have shown the characterization of doping profiles in pump probe measurements based on femtosecond ASOPS. Samples with a doped layer on top of an undoped substrate and different crystal orientations were examined. The position of the interface between the differently doped regions and the thickness of the transition region given by the Debye length could be reproduced accurately.

\section{ACKNOWLEDGMENTS}

We thank the DFG for support through the SFB 767 as well as the Ministry of Science, Research and the Arts of 
Baden-Württemberg. We would also like to thank G. Schrenk for useful discussions.

${ }^{1}$ C. Thomsen, H. T. Grahn, H. J. Maris, and J. Tauc, Phys. Rev. B 34, 4129 (1986).

${ }^{2}$ M. Trigo, A. Bruchhausen, A. Fainstein, B. Jusserand, and V. ThierryMieg, Phys. Rev. Lett. 89, 227402 (2002).

${ }^{3}$ G. W. Chern, K. H. Lin, and C. K. Sun, J. Appl. Phys. 95, 1114 (2004).

${ }^{4}$ A. Khelif, P. A. Deymier, B. Djafari-Rouhani, J. O. Vasseur, and L. Dobrzynski, J. Appl. Phys. 94, 1308 (2003).

${ }^{5}$ B. C. Daly, N. C. R. Holme, T. Buma, C. Branciard, T. B. Norris, D. M. Tennant, J. A. Taylor, and S. Pau, Appl. Phys. Lett. 84, 5180 (2004)

${ }^{6}$ K. H. Lin, C. T. Yu, S. Z. Sun, H. P. Chen, C. C. Pan, J. I. Chyi, S. W. Huang, and P. C. Li, Appl. Phys. Lett. 89, 043106 (2006).

${ }^{7}$ G. Tas, J. J. Loomis, H. J. Maris, A. A. Bailes III, and L. E. Seiberling, Appl. Phys. Lett. 72, 2235 (1998).

${ }^{8}$ Y. Ezzahri, S. Grauby, S. Dilhaire, J. M. Rampnoux, and W. Claeys, J. Appl. Phys. 101, 013705 (2007)
${ }^{9}$ K. H. Lin, C. T. Yu, Y. C. Wen, and C. K. Sun, Appl. Phys. Lett. 86, 093110 (2005).

${ }^{10}$ O. Matsuda, T. Tachizaki, T. Fuki, J. J. Baumberg, and O. B. Wright, Phys. Rev. B 71, 115330 (2005).

${ }^{11}$ A. Bartels, R. Cerna, C. Kistner, A. Thoma, F. Hudert, C. Janke, and T. Dekorsy, Rev. Sci. Instrum. 78, 035107 (2007)

${ }^{12}$ B. E. Sernelius, Phys. Rev. B 33, 8582 (1986).

${ }^{13}$ D. E. Hill, Phys. Rev. 133, A866 (1964).

${ }^{14}$ H. C. Casey, D. D. Sell, and K. W. Wecht, J. Appl. Phys. 46, 250 (1975).

${ }^{15}$ T. Dekorsy, T. Pfeifer, W. Kütt, and H. Kurz, Phys. Rev. B 47, 3842 (1993).

${ }^{16}$ O. B. Wright, B. Perrin, O. Matsuda, and V. E. Gusev, Phys. Rev. B 64, 081202 (2001).

${ }^{17}$ Y. C. Wen, L. C. Chou, H. H. Lin, V. Gusev, K. H. Lin, and C. K. Sun, Appl. Phys. Lett. 90, 172102 (2007).

${ }^{18}$ O. Matsuda and O. B. Wright, J. Opt. Soc. Am. B 19, 3028 (2002).

${ }^{19}$ T. Pezeril, P. Ruello, S. Gougeon, N. Chigarev, D. Mounier, J. M. Breteau, P. Picart, and V. Gusev, Phys. Rev. B 75, 174307 (2007). 